\title{
The role of Src family kinases in mediating M-CSF receptor signaling and monocytic cell survival
}

\author{
Yijie Wang, Melissa G. Piper, Clay B. Marsh
}

Department of Internal Medicine, Division of Pulmonary, Allergy, Critical Care, and Sleep Medicine, The Dorothy M. Davis Heart and Lung Research Institute, Ohio State University, Columbus, USA

Email: melissa.piper@osumc.edu, clay.marsh@,osumc.edu

Received 20 June 2012; revised 28 July 2012; accepted 10 August 2012

\begin{abstract}
Previously, we reported that M-CSF induced monocyte survival through the activation of Akt, p38 MAPK and Erk1/2 kinases. Here, we found that Src family kinases were upstream of these kinases and played a central role in regulating M-CSF-induced monocyte survival. We observed that M-CSF promoted c-Src activation in monocytes and MDMs in a time-dependent manner. Src inhibitors reduced M-CSF-mediated phosphorylation of the M-CSF receptor (MCSFR), Akt, Erk1/2, and p38 MAPK. We also observed that Src directly phosphorylated the M-CSFR. Notably, the inhibitors blocked phosphorylation of specific tyrosine residues within the M-CSFR. We further demonstrated that the Src inhibitor, PP2, attenuated M-CSF-induced NF- $\mathrm{KB}$ activation and MCSF-induced monocyte survival. These findings indicated that Src family kinases mediate monocyte survival through the regulation of receptor phosphorylation and modulation of downstream signaling events. Thus, we predict that targeting Src family kinases may have therapeutic implication in inflammatory diseases.
\end{abstract}

Keywords: Monocyte/Macrophage; M-CSF Receptor; Src Family Kinases; Cell Survival

\section{INTRODUCTION}

Monocytes are derived from bone marrow progenitor cells. Once released into circulation, a monocyte's lifespan is $24-48$ hours [1]. However, when a monocyte encounters specific growth factors, inflammatory cytokines or bacterial components, the monocyte enters the tissue and differentiates into a specialized tissue macrophage [2,3]. Macrophage colony-stimulating factor (MCSF) is important in promoting maturation of monocytes to macrophages as well as the survival of both cell types $[4,5]$. Survival of monocytes and macrophages occurs through the activation of Akt, Erk, p38 and JNK [6-8]. Previously, we reported that M-CSF activates Akt by suppressing caspase-3 and caspase- 9 activity [5].

M-CSF mediates the proliferation of monocyte precursors and their differentiation into mature phagocytes through its interactions with the cell surface M-CSF receptor (M-CSFR) [3]. The M-CSFR is a disulfide-linked homodimer, encoded by c-Fms proto-oncogene [9]. The M-CSFR is a member of the receptor tyrosine kinase (RTK) family, which includes platelet-derived growth factor (PDGF) receptor, c-Kit and Flt3/Flt2 receptors [10]. The M-CSFR is comprised of an Ig-like extracellular domain, juxtamembrane domain, and important intracellular domains required for its catalytic activity including ATP binding domain, kinase insert domain, and major kinase domain [11]. Upon M-CSF stimulation, the human M-CSFR undergoes dimerization and autophosphorylation of specific five tyrosine residues $(561,699$, 723,809 and 823 ) as well as ubiquitination leading to its degradation and limitation of its intracellular signaling. Phosphorylated receptor tyrosine residues are docking sites for adaptor and signaling molecules, such as Src family kinases, PI3-kinase/Akt and Ras/MAPK leading to the induction of a cascade of signaling events resulting in cell differentiation, proliferation and survival (see reviews $[12,13])$.

Src protein tyrosine kinase (PTK) family members belong to the non-receptor tyrosine kinases and are implicated in multiple signaling pathways that regulate cellular growth, migration, differentiation and survival (see review [14]). There are eight Src family kinases in vertebrates including Blk, Fyn, Fgr, Hck, Lck, Lyn, Src and Yes. Among these kinases, Hck, Lyn and Fgr are restricted to myeloid cells and B-lymphocytes, while c-Src, Yes and Fyn are ubiquitously expressed (see review [15]). All Src family kinase members possess an N-terminal unique region, a Src homology 3 (SH3) domain which binds proline rich motifs, a Src homology 2 (SH2) domain, which interacts with phosphotyrosine motifs, and a kinase domain (see review [16]). Src family kinases act 
in either a positive or a negative fashion. The Src family kinases interact with the human M-CSFR at $\mathrm{Tyr}^{561}$ residue (mouse $\mathrm{Tyr}^{559}$ ) and $\mathrm{Tyr}^{809}$ residue (mouse $\mathrm{Tyr}^{807}$ ) (see review [12]). The Src family kinase member, c-Src, is one of the most studied member. Notably, this $60 \mathrm{KDa}$ protein has two phosphorylation sites, which positively and negatively regulates its activity to ultimately regulate signal transduction pathways. Phosphorylation of $\mathrm{Tyr}^{416}$ activates c-Src kinase activity, while $\mathrm{Tyr}^{527}$ phosphorylation leads to inactivation of the kinase [17].

We previously reported that M-CSF induces the activation of Erk1/2, Akt, and p38 MAPK to regulate monocyte survival $[18,19]$. We therefore postulated that $\mathrm{M}$-CSF induces these signaling molecules and perhaps proximally through Src family kinase activation. We observed that M-CSF induced c-Src activation in monocytes. We also found that c-Src induced M-CSFR phosphorylation. Furthermore, Src family kinase inhibition reduced M-CSF-induced receptor phosphorylation as well as downstream activation events, including Akt, Erk1/2, and p38 MAPK phosphorylation resulting in a decrease in cell survival and NF-kB activation. Thus, our data indicated that members of the Src family kinases serve as a critical regulatory factor in M-CSF-mediated monocyte survival.

\section{MATERIALS AND METHODS}

\subsection{Reagents}

Endotoxin-free RPMI-1640 and PBS were purchased from Mediatec (Herndon, VA) and endotoxin-free FBS was from Atlanta Biotec (Lawrenceville, GA). Recombinant human M-CSF was purchased from R\&D Systems (Minneapolis, MN). The Src kinase inhibitor, pyrrolopyrimidine (PP2); the inactive analog, PP3 and the Src family inhibitor, SU6656 were obtained from EMD Chemicals (Gibbstown, NJ). Phospho-specific-Src Tyr ${ }^{416}$, global phospho-tyrosine (P-Tyr-100), phospho-Akt $\left(\mathrm{Ser}^{473}\right.$ ) $\left.\mathrm{Thr}^{308}\right)$, phospho-Erk1/2 $\left(\mathrm{Thr}^{202} / \mathrm{Tyr}^{204}\right)$ and phospho-p38 $\left(\mathrm{Thr}^{180} / \mathrm{Tyr}^{182}\right)$ antibodies were purchased from Cell Signaling Technology (Beverly, MA). Phospho-M-CSFR $\mathrm{Tyr}^{699}, \mathrm{Tyr}^{723}$ and $\mathrm{Tyr}^{809}$ antibodies were obtained from Cell Signaling Technology while phospho-M-CSFR $\mathrm{Tyr}^{561}$ antibody was purchased from Abcam (Cambridge, MA). M-CSFR, c-Src, Akt1 and Erk2 antibodies were obtained from Santa Cruz Biotech (Santa Cruz, CA). Recombinant full length active c-Src was obtained from Millipore (Billerica, MA). All other reagents were purchased from Sigma-Aldrich (St. Louis, MO) unless indicated otherwise.

\subsection{Cell Lines}

Murine NIH-3T3 fibroblasts stably expressing the human M-CSFR (c-Fms) and designated 3T3/fms were main- tained in DMEM supplemented with 10\% FBS and 1\% antibiotic-antimycotic (PSA: $1000 \mathrm{U} / \mathrm{ml}$ penicillin G sodium, $1000 \mu \mathrm{g} / \mathrm{ml}$ streptomycin sulfate, and $250 \mathrm{ng} / \mathrm{ml}$ amphotericin B) as described previously [20]. Prior to M-CSF stimulation, cells were serum starved in DMEM media on ice for $4-6$ hours $[8,20]$.

\subsection{Purification of Peripheral Blood Monocytes and the Generation of Monocyte-Derived Macrophages (MDMs)}

Peripheral blood monocytes were isolated from buffy coats obtained from the American Red Cross and purified by positive selection using CD14 Monocyte Isolation Kit from Miltenyi Biotech (Auburn, CA) as described previously [19]. The purity of the monocyte preparation was confirmed by flow cytometry (LSRII; BD BioSciences, San Jose, CA). The CD14 $4^{+}$isolation resulted in $97 \% \pm 3 \%$ purity. After isolation, monocytes were resuspended at $1-10 \times 10^{6}$ cells $/ \mathrm{ml}$ in RPMI-1640 medium supplemented with $10 \% \mathrm{FBS}, 10 \mu \mathrm{g} / \mathrm{ml}$ polymyxin $\mathrm{B}$ and $20 \mathrm{ng} / \mathrm{ml}$ recombinant human M-CSF then cultured overnight. The next day, cells were washed and serum starved in RPMI-1640 medium for 2 hours. Prior to M-CSF $(100 \mathrm{ng} / \mathrm{ml})$ stimulation, the cells were treated with the Src inhibitors, SU6656 or PP2, or the control inactive analog, PP3 as indicated. Since monocytes cultured for 24 hours in M-CSF are not fully differentiated macrophages, we refer to these cells as monocytes throughout this paper.

To obtain monocyte-derived macrophages (MDMs), freshly isolated monocytes were incubated in RPMI-1640 medium containing $10 \% \mathrm{FBS}, 10 \mu \mathrm{g} / \mathrm{ml}$ polymyxin $\mathrm{B}$ and $20 \mathrm{ng} / \mathrm{ml} \mathrm{M}-\mathrm{CSF}$ in a $37^{\circ} \mathrm{C} \mathrm{CO}_{2}$ incubator for 5 - 6 days. $\mathrm{M}$-CSF was replenished and maintained in the culture by the addition of $20 \mathrm{ng} / \mathrm{ml}$ of M-CSF every other day. Differentiated MDMs were serum-starved overnight at $37^{\circ} \mathrm{C}$ before being re-stimulated with M-CSF $[19,20]$.

Prior to stimulation, monocytes were serum starved in RPMI-1640 medium at $37^{\circ} \mathrm{C}$ incubator for two hours. MDMs were serum starved at $37^{\circ} \mathrm{C}$ in a $\mathrm{CO}_{2}$ incubator overnight. The next day, the media was replaced and the cells were serum starved for another two hours prior to stimulation.

\subsection{Immunoprecipitation and Immunoblotting}

Monocytes or MDMs were lysed on ice for 15 minutes in $1 \times$ lysis buffer (Cell Signaling Technology) containing protease inhibitor cocktail III (EMD Chemicals). Lysates were cleared of insoluble material then the protein concentration was determined by BioRad DC protein kit (BioRad, Hercules, CA). The samples were separated on $10 \%$ Bis-Tris SDS-PAGE gel with MOPS running buffer, transferred to a nitrocellulose membrane, probed with the 
indicated antibodies, and detected by ECL (Amersham Biosciences, Piscataway, NJ). The ECL signal was quantified using the Quantity One densitometry program (BioRad). Phosphorylation data was normalized to total protein controls or $\beta$-actin and expressed as fold-change from non-stimulated samples.

\subsection{In Vitro Kinase Assay}

After serum starvation, $3 \mathrm{~T} 3 / \mathrm{fms}$ cells were lysed in cell lysis buffer and equal amount of protein $(0.8-1 \mathrm{mg})$ was immunoprecipitated with irrelevant antibody control or M-CSFR antibody overnight at $4^{\circ} \mathrm{C}$. The immune complexes were then incubated with protein $\mathrm{G}$ beads (Life Technologies, Grand Island, NY) at $4^{\circ} \mathrm{C}$ for 1 hour. The beads were washed three times in ice-cold lysis buffer, then twice in ice-cold kinase buffer containing $25 \mathrm{mM}$ Tris- $\mathrm{HCl}$ (pH 7.5), $5 \mathrm{mM}$ beta-glycerophosphate, $2 \mathrm{mM}$ dithiothreitol (DTT), $0.1 \mathrm{mM} \mathrm{Na}_{3} \mathrm{VO}_{4}$ and $10 \mathrm{mM} \mathrm{MgCl}_{2}$. The beads were pre-incubated in $20 \mu \mathrm{l}$ kinase buffer with $10 \mu \mathrm{m}$ of PP2, PP3 or SU6656 at $37^{\circ} \mathrm{C}$ for 30 minutes prior to the addition of $100 \mu \mathrm{M}$ ATP, $20 \mathrm{ng}$ of recombinant active c-Src or both then incubated at $30^{\circ} \mathrm{C}$ for an additional 30 minutes. The reactions were subjected to SDS-PAGE analysis on a $10 \%$ SDS polyacrylamide gel. After electrophoresis, proteins were transferred onto a nitrocellulose membrane, probed with phosphotyrosine antibodies, and reblotted with M-CSFR antibodies. MCSFR phosphorylation was quantified by comparing band densitometry of phospho-M-CSFR and normalizing these values to the total M-CSFR signals in the respective lanes.

\subsection{Caspase-3 Analysis}

Caspase-3 activity assay was described previously [21]. Briefly, monocytes $\left(5 \times 10^{6}\right.$ cell/condition $)$ were treated with inhibitors for 30 minutes prior to the addition of M-CSF $(100 \mathrm{ng} / \mathrm{ml})$ then incubated overnight at $37^{\circ} \mathrm{C}$. The next day, both the suspension and adherent cells were collected and caspase-3 activity was analyzed using the fluorogenic substrate Ac-DEVD-AMC (N-acetyl(Asp-Glu-Val-Asp)-(7-amino-4-methylcoumarin)) (EMD Chemicals) as measured with Cytofluor 4000 fluorometer (Perseptive, Framingham, MA). The linear change of the fluorescence of hydrolyzed free AMC was used to calculate caspase-3 activity. Protein concentration was measured using BCA protein assay kit (Therma Scientific, Rockford, IL). Caspase-3 activity is presented as the relative fold-increase in the individual sample readout over M-CSF-stimulated sample readout per total protein and are expressed as the mean $\pm \mathrm{SEM}$.

\subsection{Annexin V/PI Apoptosis Assay}

Annexin V/PI cell apoptosis assay was performed as described previously [19] using the Annexin V-FITC apoptosis detection kit (BD PharMingen, San Diego, CA). Briefly, human monocytes $\left(5 \times 10^{6}\right)$ were incubated with inhibitors for 30 minutes in serum free X-vivo (Lonza Walkersville Inc., Walkersville, MD) before the addition of M-CSF $(100 \mathrm{ng} / \mathrm{ml})$ then incubated overnight at $37^{\circ} \mathrm{C}$. The cells were removed from the culture dish using Accutase (eBioscience, San Diego, CA), then stained with Annexin V-FITC and PI. The cells were analyzed by flow cytometry. Annexin V-FITC and PI double positive cells were considered apoptotic cells for the statistical analysis.

\subsection{Transfection of Monocytes and NF-кB Activity Assay}

Transient transfection of primary human monocytes was performed as described previously [19] using the Amaxa Nucleofector system (Lonza, Walkersville, MD). The

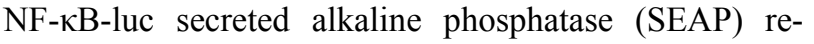
porter assay was performed as described previously [20]. Briefly, monocytes $\left(15 \times 10^{6}\right)$ isolated from buffy coats were resuspended in Monocyte Transfection Solution (Lonza) containing $2 \mu \mathrm{g}$ of NF-kB-Luc construct and transfected using the Amaxa program Y-01. The cells were immediately washed in warm X-vivo medium and plated in triplicate in 24-well plates. After an hour, the monocytes were incubated with inhibitors for 30 minutes in X-vivo medium before addition of M-CSF $(100 \mathrm{ng} / \mathrm{ml})$ then cultured overnight at $37^{\circ} \mathrm{C}$. Luciferase production was measured with the Luciferase Assay System using a Luminometer (Promega, Madison, WI). Data are presented as the relative fold increase in luciferase production over nonstimulated mock transfected samples and are expressed as the mean $\pm \mathrm{SEM}$.

\subsection{Statistical Analysis}

All data are expressed as the mean \pm SEM derived from at least three independent studies. Statistical analysis was performed with SPSS17 software (SPSS Inc. Chicago, IL) by using independent sample t-test. Statistical significance was defined as $\mathrm{p}<0.05$.

\section{RESULTS}

\subsection{M-CSF Induced Src Activation in Human Monocytes and MDM}

In this study, we hypothesized that Src family kinases are involved in M-CSF-regulated survival pathways in both monocytes and macrophages. Since Src family kinases are tyrosine phosphorylated following M-CSF stimulation in fibroblast cell lines overexpressing c-Fms [22], we first examined whether M-CSF directly activated c-Src as proximal kinase, in primary human monocytes 
or MDMs. Therefore, cells were treated in the presence or absence of M-CSF at the indicated time and western blot analysis was performed to detect active phosphoc-Src $\left(\mathrm{Tyr}^{416}\right)$. In both primary monocytes and MDMs, phosphorylated c-Src $\left(\mathrm{Tyr}^{416}\right)$ was apparent after 2 minutes of M-CSF stimulation (Figures 1(a)-(d)). Significant increase of c-Src phosphorylation was observed after 5 and 10 minutes of M-CSF stimulation in monocytes, while c-Src phosphorylation was significantly increased within 2 minutes of M-CSF stimulation in MDMs. The phosphorylation then returned to basal levels after 20 minutes of stimulation in both monocytes and MDMs.

\subsection{Src Kinase Inhibitors Reduced M-CSF-Induced M-CSFR Phosphorylation}

Previous research reported that the PDGF receptor is a substrate for Src kinases [23]. Since both the M-CSFR and PDGFR are in the same tyrosine kinase receptor

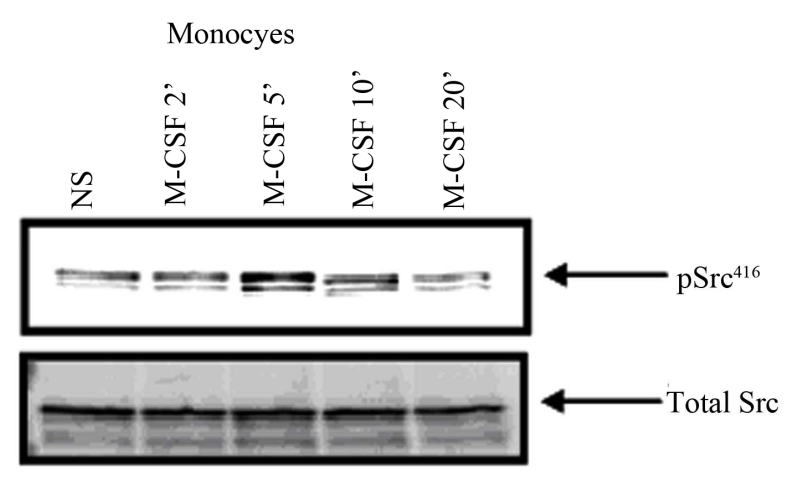

(a)

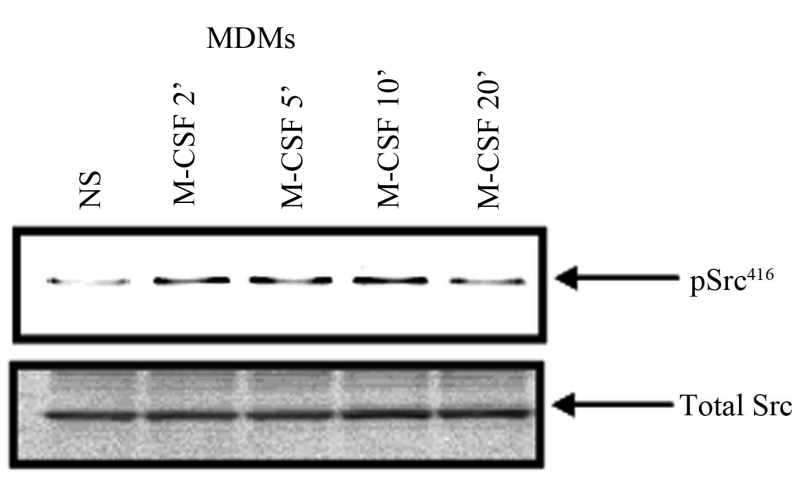

(c) family [24], we were interested in determining whether the M-CSFR is also a substrate for Src family kinases. To test this hypothesis, we treated the cells with Src kinase inhibitors, PP2 or SU6656, or an inactive analogue, PP3, prior to M-CSF stimulation and then examined M-CSFR phosphorylation. Since M-CSFR is expressed at very low levels in monocytes and is up-regulated during macrophage differentiation [11], we utilized MDMs to examine M-CSFR phosphorylation. As shown in Figures 2(a) and (b), tyrosine phosphorylation of the M-CSFR was enhanced in response to M-CSF, but was significantly reduced by the PP2 inhibitor, but not inactive analogue PP3. Similar reduction in receptor phosphorylation was observed in cells treated with M-CSF and the SU6656 inhibitor. Importantly, using trypan blue exclusion, we did not detect non-specific cytotoxicity from the inhibitors (data not shown). These data suggested that the M-CSFR is a substrate for Src family kinases.

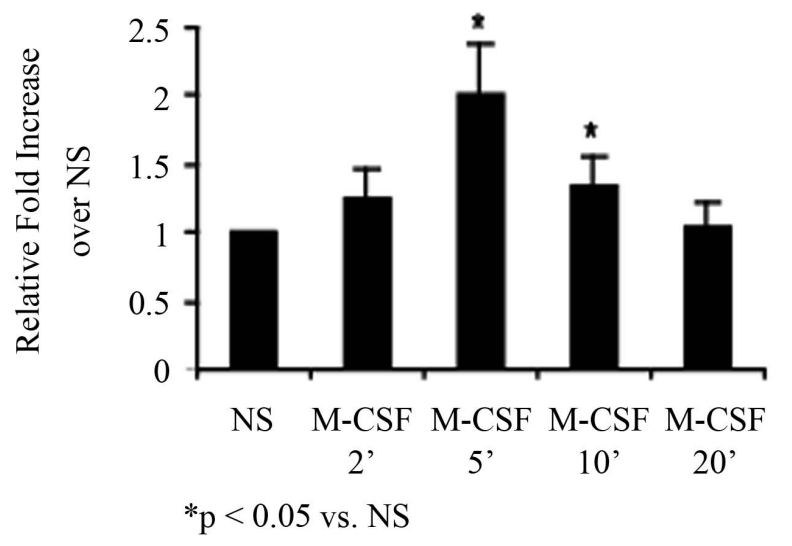

(b)

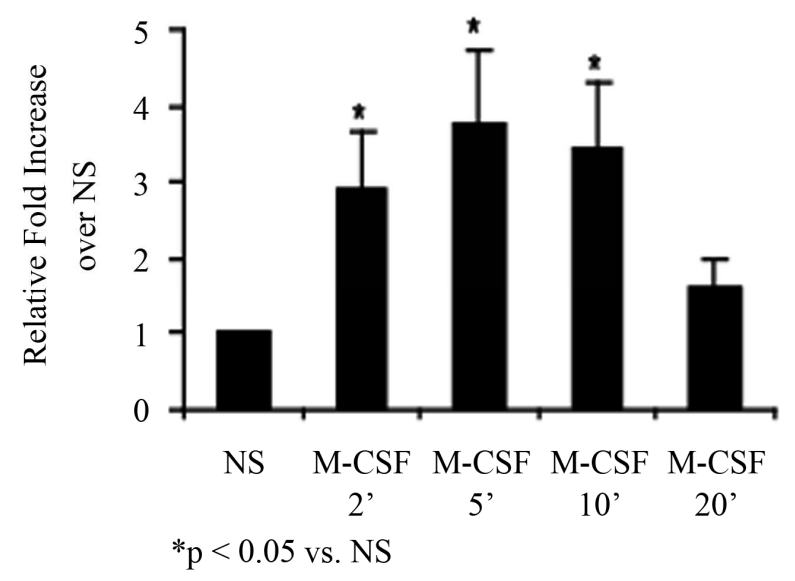

(d)

Figure 1. M-CSF increased c-Src phosphorylation. (a) and (b) Freshly isolated human monocytes $\left(10 \times 10^{6} / \mathrm{condition}\right)$ or (c) and (d) MDM $\left(10 \times 10^{6} /\right.$ condition) were stimulated with $100 \mathrm{ng} / \mathrm{ml} \mathrm{M-CSF}$ for the indicated times. Cells were lysed and c-Src phosphorylation was measured by western blot analysis using an antibody recognizing the phospho-Src Tyr ${ }^{416}$. Densitometry quantification of the blots comparing phosphorylated c-Src $\mathrm{Tyr}^{416}$ to total c-Src (lower panel). Data are expressed as the mean \pm SEM from three independent donors ( $\mathrm{p}<0.05)$. 


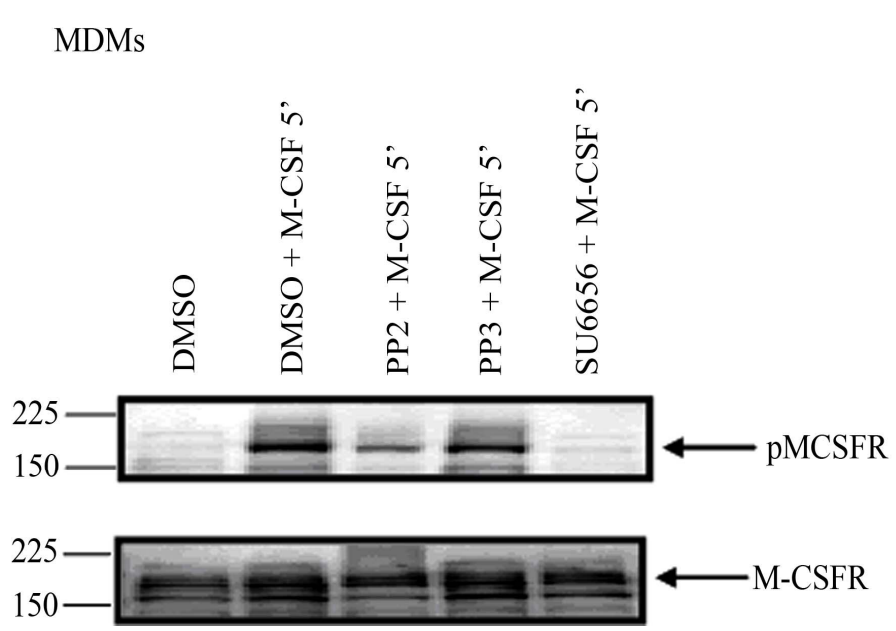

(a)

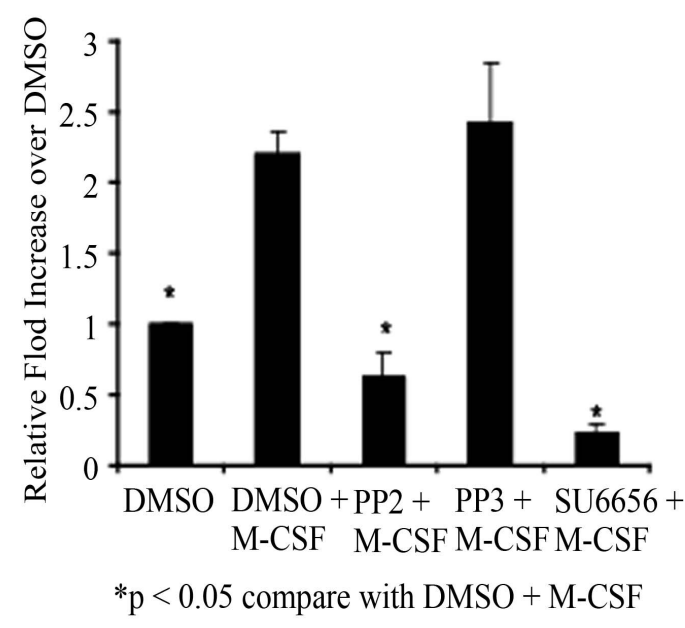

(b)

Figure 2. Src kinase inhibition reduces M-CSFR phosphorylation in primary MDMs. (a) MDMs $\left(10 \times 10^{6} /\right.$ condition $)$ were serum starved overnight and pretreated with DMSO solvent, $10 \mu \mathrm{M}$ of PP2, PP3 or SU6656 for 30 minutes prior to stimulation with 100 $\mathrm{ng} / \mathrm{ml}$ M-CSF for 5 minutes. Proteins were extracted from the cells and equal amount of protein was resolved on a $10 \%$ SDS-PAGE gel, and immunoblotted with phosphotyrosine antibody. The blots were then immunoblotted with total M-CSFR antibody; (b) M-CSFR phosphorylation was quantified by densitometry and the phospho-M-CSFR band was compared to total M-CSFR in the respective lanes. Data are expressed as the mean \pm SEM from three independent donors $\left({ }^{*} \mathrm{p}<0.05\right.$ compare with DMSO $+\mathrm{M}-\mathrm{CSF}$ treated samples).

Since global tyrosine phosphorylation of the M-CSFR was reduced in the presence of M-CSF and Src kinase inhibitors, we examined whether this inhibition affected a specific tyrosine residue. To analysis the phosphorylation status of specific tyrosine residues, we utilized the 3T3/fms cell line to ensure sufficient M-CSFR levels to be visualized by western blot analysis. The $3 \mathrm{~T} 3 / \mathrm{fms}$ cells were pretreated with either PP2 or SU6656 prior to stimulation with M-CSF then divided among separate gels for western blot analysis with each phospho-tyrosine antibody as indicated. Similar to MDMs, Src inhibition reduced M-CSF mediated phosphorylation in $3 \mathrm{~T} 3 / \mathrm{fms}$ cells (Figure 3(a)). In the presence of M-CSF and either Src kinase inhibitors, PP2 or SU6656, as indicated, reduced M-CSFR tyrosine phosphorylation at residues 699, 723 and 809 was apparent with (Figure 3(a)) while $\mathrm{Tyr}^{561}$ was relatively unchanged. Notably, $\mathrm{Tyr}^{561}$ of the M-CSFR is the Src docking site (see review [13]).

\subsection{The M-CSFR Is a Substrate for c-Src}

To further confirm that the M-CSFR can be directly phosphorylated by Src kinases, we performed an in vitro kinase activity assay. To perform the kinase assay, the immunoprecipitated M-CSFR from 3T3/fms cells was incubated with or without recombinant active c-Src protein (Figures 3(b) and (c)). Due to intrinsic kinase activity of the receptor, we detected M-CSFR phosphorylation in the presence of ATP alone (Figure 3(c), ATP) or ATP and the inactive analog PP3 (Figure 3(b), PP3 + ATP). In samples treated with both recombinant active c-Src and ATP (PP3 + Src + ATP or Src + ATP), we observed increased M-CSFR phosphorylation compared to samples treated with ATP only (ATP or PP3 + ATP) (Figures 3(b) and (c)). Interestingly, there was a $54 \% \pm 6 \%$ and $42 \% \pm 18 \%$ decrease in M-CSFR phosphorylation in the presence ATP alone with either PP2 or SU6656, respectively. This observation reflects the ability of the inhibitors to non-selectively interfere with intrinsic kinase activity of the M-CSFR. Using Src kinase inhibitors in the presence of ATP and recombinant active c-Src, we observed further reduction in M-CSFR phosphorylation compared to the control samples. Notably, there was a $75 \% \pm 3 \%$ reduction in receptor phosphorylation comparing PP2 + c-Src + ATP samples to PP3 + c-Src + ATP samples (Figure 3(b)). Likewise, there was an $82 \% \pm$ $8 \%$ reduction in M-CSFR phosphorylation in SU6656 + c-Src + ATP samples compared to c-Src + ATP samples (Figure 3(c)). As expected, treatment with the inhibitors with or without recombinant active c-Src in the absence of ATP did not induce M-CSFR phosphorylation. Thus, our data indicated that the M-CSFR is a substrate for Src family kinases.

\subsection{Inhibition of Src Kinases Reduce M-CSF-Induced Intracellular Signaling and NF-кB Activity in Human Monocyte and MDM}

Our previous work identified that M-CSF and reactive oxygen species (ROS) induces Akt, Erk1/2 and p38 MAPK phosphorylation in human monocytes $[18,19]$. 


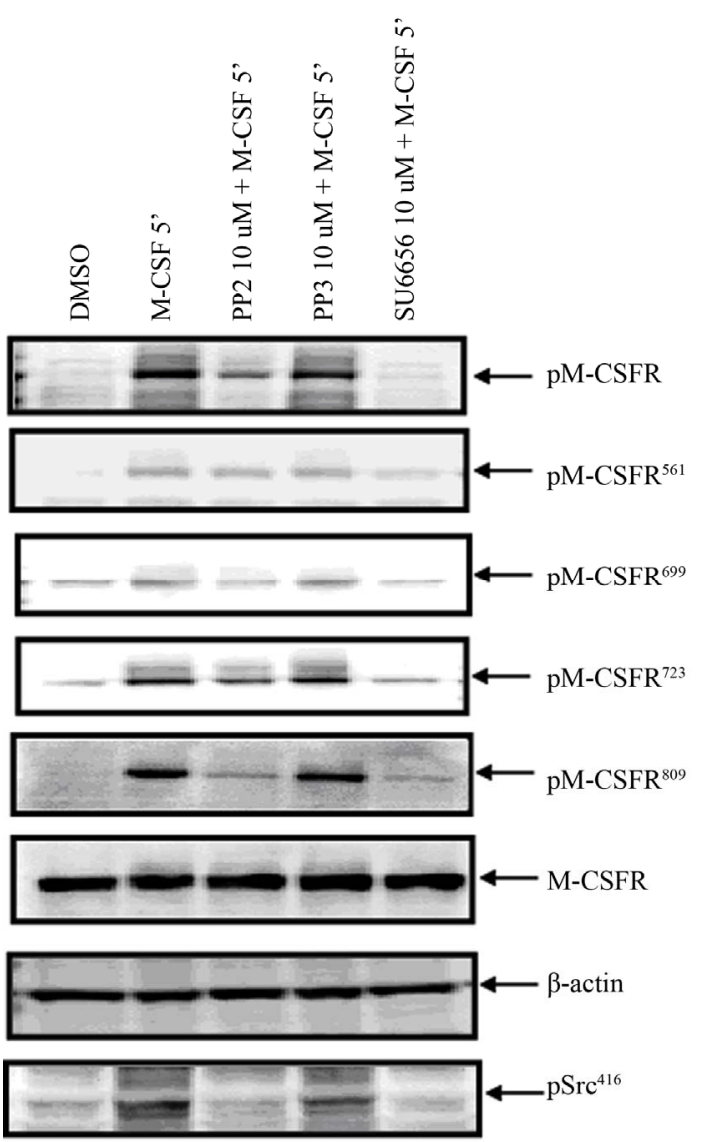

(a)

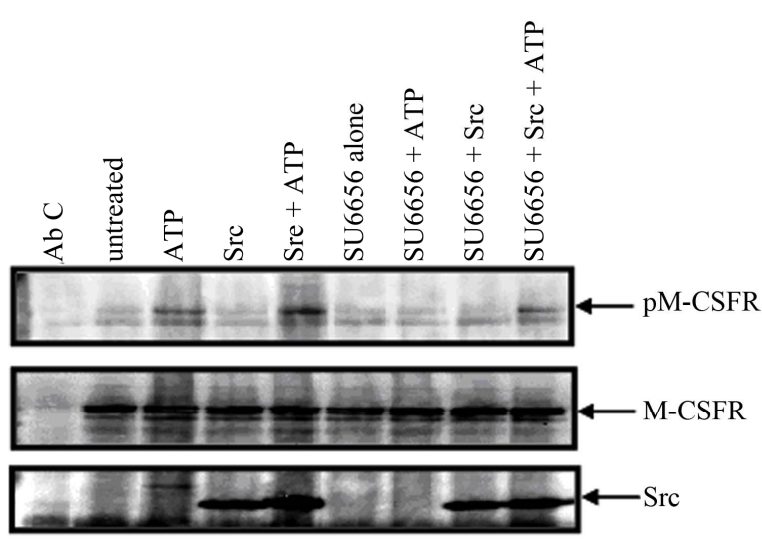

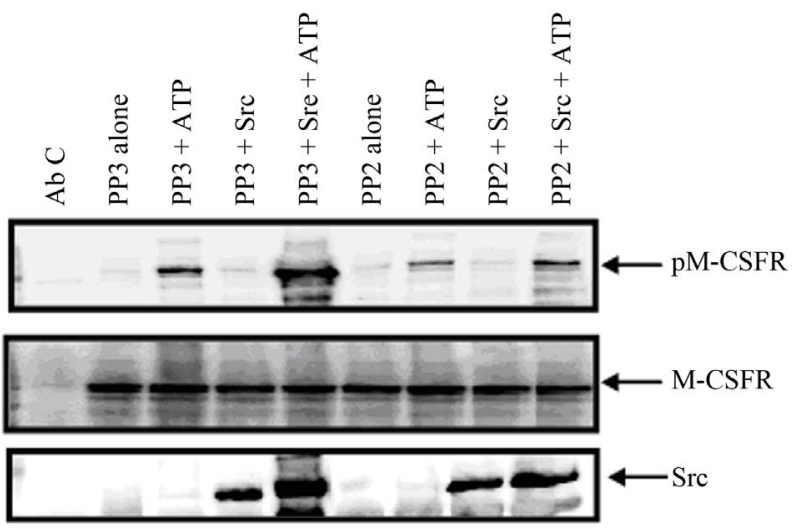

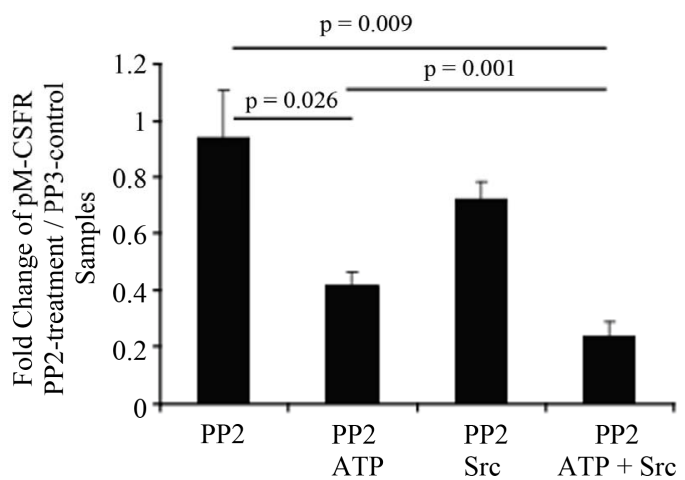

(b)

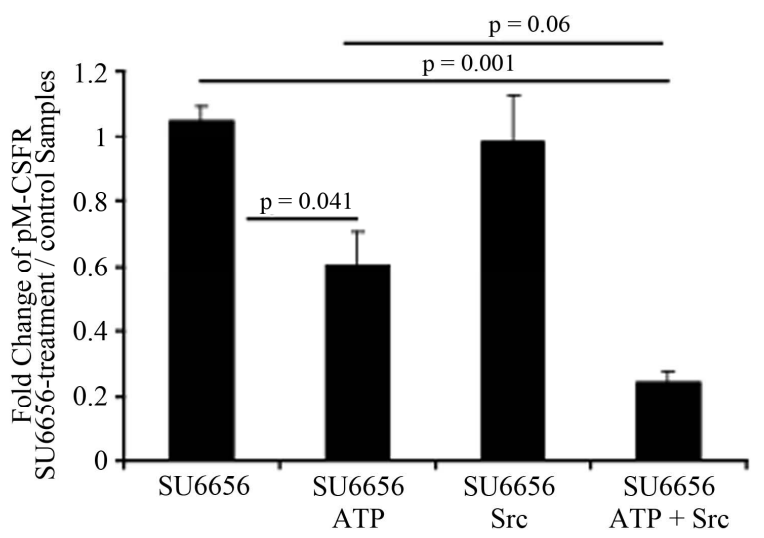

(c)

Figure 3. The M-CSFR is a substrate for Src kinases. (a) $3 \mathrm{~T} 3 / \mathrm{fms}$ cells $\left(5 \times 10^{6} /\right.$ condition) were serum starved overnight and then pretreated with DMSO solvent, $10 \mu \mathrm{M}$ of PP2, PP3 or SU6656 for 30 minutes prior to stimulation with $100 \mathrm{ng} / \mathrm{ml}$ M-CSF for 5 minutes. Equal amount of protein was separated on multiple 10\% SDS-PAGE gels and immunoblotted with phospho-tyrosine antibody or specific M-CSFR phospho-tyrosine antibodies as indicated. To confirm equal loading, the blots were immunoblotted with total M-CSFR antibody and $\beta$-actin antibody; (b) and (c) In vitro kinase assay was performed using protein lysates from 3T3/fms cells treated with or without Src kinase inhibitors PP2 (b) and SU6656 (c) that were immunoprecipitated with the M-CSFR antibody. The M-CSFR immune complexes were incubated with ATP or c-Src as indicated then subjected to western blot analysis with phospho-tyrosine antibody to measure to M-CSFR phosphorylation. M-CSFR phosphorylation was quantified by densitometry and normalized to the total M-CSFR signals in the respective lanes. The fold decrease of PP2-treated samples compared to the corresponding PP3-treated samples (Figure 3(b), lower panel) or the SU6656-treated samples over the corresponding control samples (Figure 3(c), lower panel) is shown. Data are expressed as the mean \pm SEM from three independent experiments. 
Therefore, we next examined the role of Src family kinases in M-CSFR-mediated activation of these signaling molecules in monocytes and MDMs. Since these cells contain multiple Src kinase members, we used the general Src tyrosine kinase inhibitor, PP2 instead of trying to use multiple siRNAs. Monocytes and MDMs were treated with either PP2 or the control, PP3, for 30 minutes prior to M-CSF stimulation and then phosphorylation of the signaling molecules was examined. As predicted, PP2, but not the inactive analogue PP3, significantly reduced M-CSF-induced phosphorylation of Akt, Erk $1 / 2$ and p38 in human monocytes (Figures 4(a) and (b)) and MDMs (Figures 4(c) and (d)).

\subsection{Inhibition of Src Kinases Reduce M-CSF-Induced NF-KB Activity in Human Monocyte}

Since NF-KB activity is important in monocyte survival and function [19,20], we next examined the role of Src family kinases on M-CSF-induced NF- $\mathrm{KB}$ activation in monocytes. To examine NF- $\mathrm{kB}$ activity, we utilized a luciferase reporter system. Monocytes transiently transfected with a NF-KB-Luc construct were cultured in the absence or presence of Src kinase inhibitors with M-CSF.

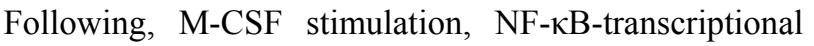
activity was increased in monocytes (Figure 5). Notably, the kinase inhibitor PP2 reduced M-CSF-induced NF- $\mathrm{kB}$ activity $(\mathrm{p}<0.05)$.

\subsection{Activation of Src Kinases Is Important in M-CSF Mediated Survival in Monocytes}

Previously, our laboratory reported that the activation of Akt, Erk $1 / 2$ and NF-kB is important in monocyte survival $[5,7]$. Since inhibition of Src family kinases prevented M-CSF-induced receptor phosphorylation as well as downstream activation of signaling path ways in

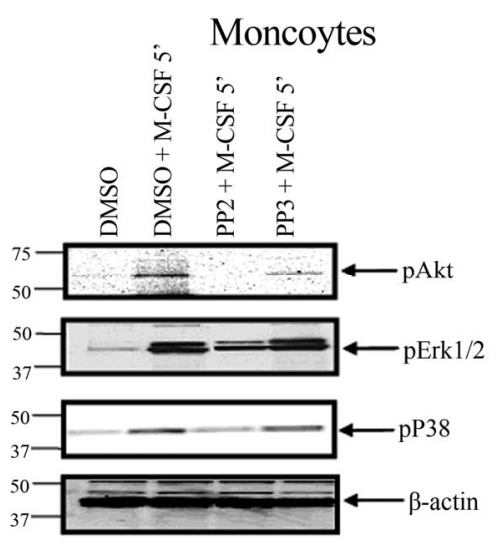

(a)

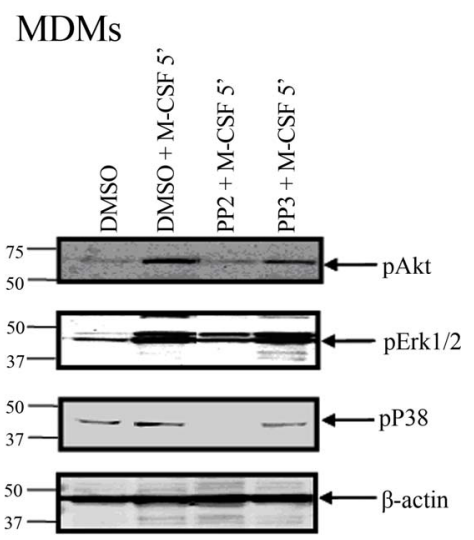

(c)

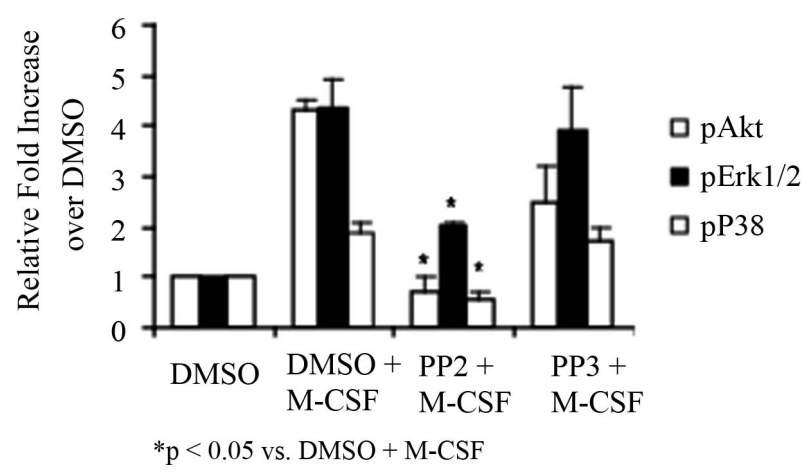

(b)

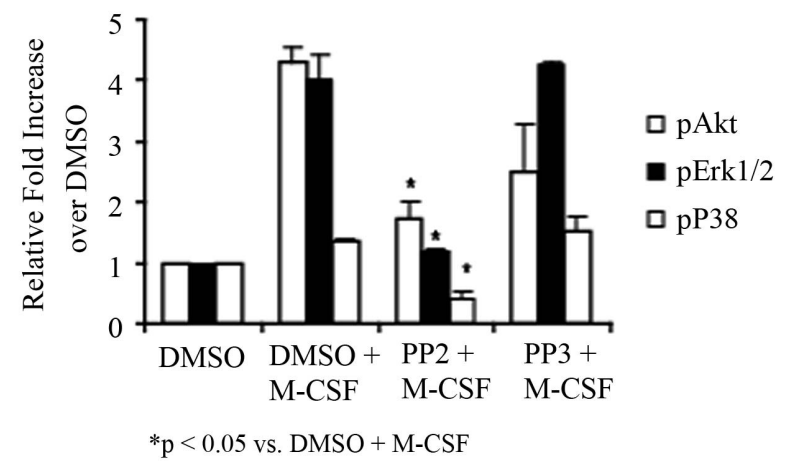

(d)

Figure 4. Inhibition of Src kinases suppresses M-CSF-induced Akt, Erk1/2 and p38 phosphorylation in primary human monocytes and MDMs. Freshly isolated monocytes (a) or MDMs (c) were incubated with DMSO solvent, PP2 or PP3 $(10 \mu \mathrm{M})$ for 30 minutes before stimulation with M-CSF at $37^{\circ} \mathrm{C}$ for 5 minutes. Cells were lysed and equal amount of protein was resolved using a 10\% SDS-PAGE gel then immunoblotted with the indicated antibodies. Equal loading was confirmed by reblotting the membranes with $\beta$-actin antibody. Phosphorylation levels were quantified by comparing the densitometry of the phosphorylated protein bands to $\beta$-actin. The data are presented as the mean \pm SEM from three independent donors $\left({ }^{*} \mathrm{p}<0.05\right)$. 


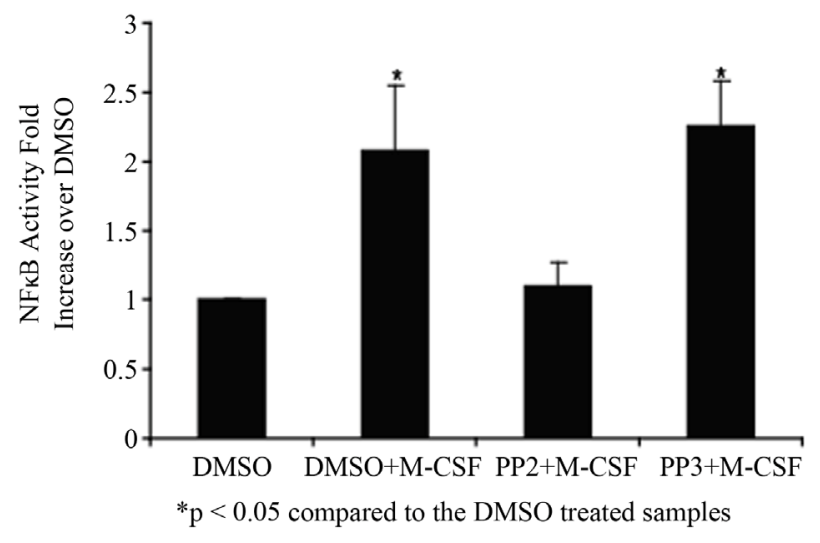

Figure 5. Src kinase inhibition decreases M-CSF-induced NF$\kappa \mathrm{B}$ activity. Freshly isolated monocytes $\left(15 \times 10^{6}\right)$ were transfected with $2 \mu \mathrm{g}$ of NF- $\kappa \mathrm{B}$-Luc construct or eGFP control construct. After an hour, the monocytes were incubated with inhibitors for 30 minutes in X-vivo medium before addition of M-CSF $(100 \mathrm{ng} / \mathrm{ml})$ then cultured overnight at $37^{\circ} \mathrm{C}$. Cells were lysed and luciferase activity was measured using a luminometer to detect luciferase activity. Data are presented as the relative fold-increase in non-stimulated mock transfected samples and are expressed as the mean \pm SEM from three independent donors done in triplicate $\left(^{*} \mathrm{p}<0.05\right.$ compared with DMSO alone treated samples).

monocytes, we next examined if cell survival was altered by Src family kinase inhibition. As shown in Figure 6(a), non-stimulated cells (DMSO) had high levels of caspase-3 activity compared to M-CSF-treated cells (DMSO $+\mathrm{M}-\mathrm{CSF})(\mathrm{p}<0.05)$. As expected, the Src family kinase inhibitor PP2, but not the inactive analogue PP3, induced caspase-3 activity in M-CSF-stimulated monocytes.

As an alternative method of measuring cell apoptosis, we measured Annexin V/PI staining in the treated cells by flow cytometry. Apoptotic cells were designated as Annexin V+/PI- (early apoptosis) and Annexin V+/PI+ populations (late apoptosis) while viable cells were Annexin $\mathrm{V}-/ \mathrm{PI}-$. Compared to DMSO treated cells, M-CSF protected monocytes from apoptosis, but inhibition of Src family kinases with PP2 in the presence of M-CSF significantly reduced the survival of the monocytes (Figure 6(b)). Collectively, these data showed that Src family kinases regulate M-CSF-induced survival of human monocyte survival through NF- $\mathrm{kB}$ activation as well as other pathways in monocytes.

\section{DISCUSSION}

Previously, we found M-CSF modulates monocyte survival by activating Akt, p38 MAPK, and Erk1/2 kinases $[5,18,19]$. In this study, we focused on understanding the role of Src family kinases as proximal signaling intermediaries in downstream networks activated by M-CSF in monocytes and MDMs. We found cross-talk between Src family kinases and the M-CSFR. Specifically, inhibition

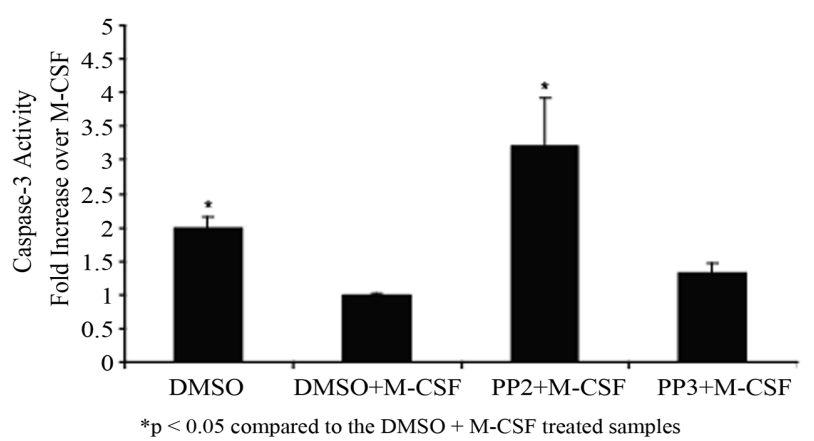

(a)

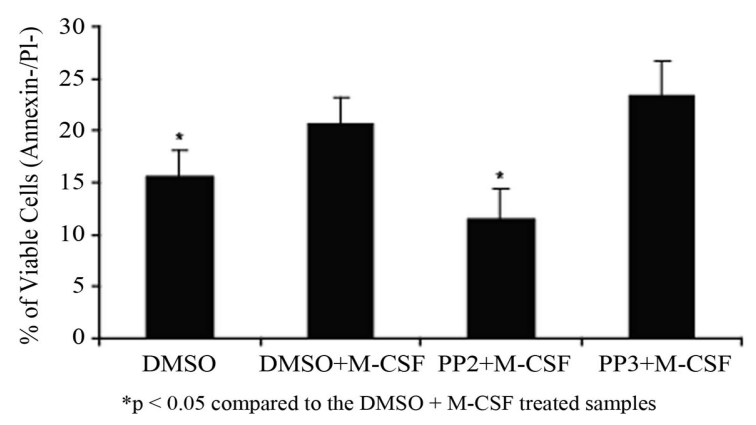

(b)

Figure 6. Src kinase inhibition decreases M-CSF-induced cell survival. Freshly isolated human monocytes $\left(5 \times 10^{6}\right)$ were pretreated with DMSO solvent, PP2 or PP3 $(10 \mu \mathrm{M})$ in RPMI 1640 medium for 30 minutes then stimulated with $100 \mathrm{ng} / \mathrm{ml}$ M-CSF overnight. (a) Cells were lysed and caspase-3 activity was measured with DEVD-AMC as substrate. Caspase-3 activity is presented as the relative fold increase compared to $\mathrm{M}$ CSF-stimulated sample (DMSO + M-CSF). Data are expressed as the mean \pm SEM from three independent donors done in triplicate $\left(^{*} \mathrm{p}<0.05\right.$ compared with DMSO + M-CSF treated sample); (b) Cells were removed from the plate using Accutase and stained with Annexin V/PI then analyzed by flow cytometry. Data are presented as percent of viable cells (Annexin $\mathrm{V}-/ \mathrm{PI}-)$ and expressed as mean \pm SEM from three independent donors ${ }^{*} \mathrm{p}<0.05$ compared to DMSO + M-CSF treated samples).

of Src family kinase activity in monocytes and MDMs reduced the activation of Akt, Erk1/2 and p38 MAPK and induced monocyte apoptosis. Moreover, inhibition of Src family kinases decreased NF- $\mathrm{KB}$ transcriptional activity after M-CSF stimulation. Our data indicate that Src family kinases play central roles in the M-CSF-mediated primary myeloid cell survival.

The well-established paradigm is M-CSF binds the M-CSFR, leading to receptor dimerization and autophosphorylation, providing docking sites for signaling molecules. Src family kinases bind the phosphorylated $\mathrm{Tyr}^{561}$ residue in human M-CSFR (or $\mathrm{Tyr}^{559}$ in the murine M-CSFR) resulting in their activation and downstream signaling events [12]. Since there are nine Src family kinase members in myeloid cells and their function are redundant, it is not feasible to use molecular and genetic 
approaches to reduce these kinases. Targeting c-Src alone with siRNA or a dominant negative construct was not effective in our hands. Thus, requiring the use of the pharmacological Src family kinase inhibitors PP2 or SU6656 to effective inhibits the activity of all Src family kinase members.

Since, we observed that M-CSFR phosphorylation was reduced by inhibition of Src kinases this suggested that Src family kinases also mediate M-CSFR phosphorylation, similar to the finding that the PDGF receptor is a substrate for Src kinases [25]. Interestingly, the mutant Y559F murine M-CSFR reduces M-CSFR phosphorylation [26], while mutations at tyrosine residues 706, 921 or 947 do not affect receptor tyrosine phosphorylation [3]. In the presence of Src inhibitors, phosphorylation of the $\mathrm{Tyr}^{561}$ of the human M-CSFR was relatively unchanged but reduced at other residues. The in vitro kinase activity assay using human M-CSFR further demonstrated that the receptor could serve as a substrate for c-Src. Similar to our findings, Galvagni and colleagues recently reported the Src-dependent phosphorylation of VEGFR-3 in endothelial cells [27]. Furthermore, PP2 reduced EGFinduced VEGFR-3 receptor phosphorylation [27]. Interestingly, ROS can activate Src kinases independent of receptor activation [28]. In turn, ROS-activated Src kinases mediate PDGF receptor phosphorylation [28]. We also observed that ROS mediate M-CSFR phosphorylation through a Src-dependent pathway (data not shown). Since, the M-CSFR belongs to the same RTK family as VEGF and PDGF receptors, it is likely this phosphorylation feedback loop is conserved among RTKs.

The role of Src family kinase activation in regulating monocyte survival and proliferation has been reported. In microglia, GM-CSF activates the Src family kinase, Hck, which is involved in the activation of PI-3K/Akt cell proliferation and survival [29]. Our laboratory reported that Lyn, another Src family kinase, negatively regulates M-CSF-induced monocyte survival by recruiting SHIP1 to the cell membrane and down regulating PI-3K/Akt activation [30]. Moreover, $\mathrm{Lyn}^{-1-}$ macrophages have increased Akt activity in response to M-CSF [30]. Consistent with these observations, we found that Src family kinases are important in M-CSF-induced activation of PI-3K/Akt and Erk pathways [10]. Most recently, Rovida and colleagues reported Src kinase inhibitors reduced M-CSF-induced Akt and Erk5 phosphorylation in human and mouse macrophage cell lines [31]. In our study, we found that pharmacological inhibition of Src family kinases reduced tyrosine phosphorylation of the MCSFR at tyrosine residues 699, 723 and 809 that provide docking sites for intracellular signaling proteins such as Grb2-SOS, PI3-Kinase and Ras that regulate cellular survival pathways in monocytes (see review [13]). Taken together, these observations indicate that Src kinases are pivotal in regulating M-CSF signaling as well as survival pathways.

In summary, this study demonstrates that Src family kinases play a central role in primary mononuclear phagocytes survival by contributing to M-CSFR activation and downstream signaling events. In conditions such as chronic inflammation where there is prolonged survival of macrophages, it is possible that targeting Src kinases to limit cell survival and tissue damage may be a beneficial therapeutic option. Current studies are underway to examine the activation of Src kinases in chronic inflammatory diseases.

\section{REFERENCES}

[1] Cline, M.J., Lehrer, R.I., Territo, M.C. and Golde, D.W. (1978) Monocytes and macrophages: Functions and diseases. Annals of Internal Medicine, 88, 78-88. http://annals.org/article. aspx? volume $=88 \&$ page $=78$

[2] Chitu, V. and Stanley, E.R. (2006) Colony-stimulating factor-1 in immunity and inflammation. Current Opinion in Immunology, 18, 39-48. doi:10.1016/j.coi.2005.11.006

[3] Yu, W., Chen, J., Xiong, Y., Pixley, F.J., Dai, X.M., Yeung, Y.G., et al. (2008) CSF-1 receptor structure/function in $\mathrm{MacCsflr}^{-1-}$ macrophages: Regulation of proliferation, differentiation, and morphology. Journal of Leukocyte Biology, 84, 852-863. doi:10.1189/jlb.0308171

[4] Praloran, V. (1991) Structure, biosynthesis and biological roles of monocyte-macrophage colony stimulating factor (CSF-1 or M-CSF). Nouvelle Revue Francaise D Hematologie, 33, 323-333.

[5] Goyal, A., Wang, Y., Graham, M.M., Doseff, A.I., Bhatt, N.Y. and Marsh, C.B. (2002) Monocyte survival factors induce Akt activation and suppress caspase-3. American Journal of Respiratory Cell and Molecular Biology, 26, 224-230.

http://ajrcmb.atsjournals.org/content/26/2/224.long

[6] Jaworowski, A., Wilson, N.J., Christy, E., Byrne, R. and Hamilton, J.A. (1999) Roles of the mitogen-activated protein kinase family in macrophage responses to colony stimulating factor-1 addition and withdrawal. Journal of Biological Chemistry, 274, 15127-15133. doi:10.1074/jbc.274.21.15127

[7] Zeigler, M.M., Doseff, A.I., Galloway, M.F., Opalek, J.M., Nowicki, P.T., Zweier, J.L., et al. (2003) Presentation of nitric oxide regulates monocyte survival through effects on caspase-9 and caspase-3 activation. Journal of Biological Chemistry, 278, 12894-12902. doi:10.1074/jbc.M213125200

[8] Kelley, T.W., Graham, M.M., Doseff, A.I., Pomerantz, R.W., Lau, S.M., Ostrowski, M.C., et al. (1999) Macrophage colony-stimulating factor promotes cell survival through Akt/protein kinase B. Journal of Biological Chemistry, 274, 26393-26398. doi:10.1074/jbc.274.37.26393

[9] Timms, J.F., Carlberg, K., Gu, H., Chen, H., Kamatkar, S., Nadler, M.J., et al. (1998) Identification of major 
binding proteins and substrates for the SH2-containing protein tyrosine phosphatase SHP-1 in macrophages. $\mathrm{Mo}$ lecular and Cellular Biology, 18, 3838-3850. http://mcb.asm.org/content/18/7/3838.full

[10] Lee, A.W. (1992) Signal transduction by the colonystimulating factor-1 receptor; comparison to other recaptor tyrosine kinases. Current Topics of Cellular Regulation, 32, 73-181.

[11] Pixley, F.J. and Stanley, E.R. (2004) CSF-1 regulation of the wandering macrophage: Complexity in action. Trends in Cell Biology, 14, 628-638. doi:10.1016/j.tcb.2004.09.016

[12] Hamilton, J.A. (1997) CSF-1 signal transduction. Journal of Leukocyte Biology, 62, 145-155. http://www.jleukbio.org/content/62/2/145.long

[13] Hunter, M., Wang, Y., Eubank, T., Baran, C., NanaSinkam, P. and Marsh, C. (2009) Survival of monocytes and macrophages and their role in health and disease. Frontiers in Bioscience, 14, 4079-4102. doi: $10.2741 / 3514$

[14] Okutani, D., Lodyga, M., Han, B. and Liu, M. (2006) Src protein tyrosine kinase family and acute inflammatory responses. American Journal of Physiology-Lung Cellular and Molecular Physiology, 291, L129-L141. doi:10.1152/ajplung.00261.2005

[15] Thomas, S.M. and Brugge, J.S. (1997) Cellular functions regulated by Src family kinases. Annual Review of Cell and Developmental Biology, 13, 513-609. doi:10.1146/annurev.cellbio.13.1.513

[16] Ingley, E. (2008) Src family kinases: Regulation of their activities, levels and identification of new pathways. Biochimica et Biophysica Acta, 1784, 56-65. doi:10.1016/j.bbapap.2007.08.012

[17] Hunter, T. (1987) A tail of two Src's: Mutatis mutandis. Cell, 49, 1-4. doi:10.1016/0092-8674(87)90745-8

[18] Bhatt, N.Y., Kelley, T.W., Khramtsov, V.V., Wang, Y., Lam, G.K., Clanton, T.L., et al. (2002) Macrophagecolony-stimulating factor-induced activation of extracellular-regulated kinase involves phosphatidylinositol 3kinase and reactive oxygen species in human monocytes. Journal of Immunology, 169, 6427-6434. http://www.jimmunol.org/content/169/11/6427.full.pdf

[19] Wang, Y., Zeigler, M.M., Lam, G.K., Hunter, M.G., Eubank, T.D., Khramtsov, V.V., et al. (2007) The role of the NADPH oxidase complex, p38 MAPK, and Akt in regulating human monocyte/macrophage survival. American Journal of Respiratory Cell and Molecular Biology, 36, 68-77. doi:10.1165/rcmb.2006-0165OC

[20] Wang, Y., Keogh, R.J., Hunter, M.G., Mitchell, C.A., Frey, R.S., Javaid, K., et al. (2004) SHIP2 is recruited to the cell membrane upon macrophage colony-stimulating factor (M-CSF) stimulation and regulates M-CSF-induced signaling. Journal of Immunology, 173, 6820-6830. http://www.jimmunol.org/content/173/11/6820.full

[21] Wang, Y., Wang, H., Piper, M.G., McMaken, S., Mo, X., Opalek, J., et al. (2010) sRAGE induces human monocyte survival and differentiation. Journal of Immunology, 185, 1822-1835. http://www.jimmunol.org/content/185/3/1822.full

\section{doi:10.4049/jimmunol.0903398}

[22] Courtneidge, S.A., Dhand, R., Pilat, D., Twamley, G.M., Waterfield, M.D. and Roussel, M.F. (1993) Activation of Src family kinases by colony stimulating factor-1, and their association with its receptor. European Molecular Biology Organization Journal, 12, 943-950.

http://www.ncbi.nlm.nih.gov/pmc/articles/PMC413295/?t ool=pubmed

[23] Hansen, K., Johnell, M., Siegbahn, A., Rorsman, C., Engstrom, U., Wernstedt, C., et al. (1996) Mutation of a Src phosphorylation site in the PDGF beta-receptor leads to increased PDGF-stimulated chemotaxis but decreased mitogenesis. European Molecular Biology Organization Journal, 15, 5299-5313.

http://www.ncbi.nlm.nih.gov/pmc/articles/PMC452274/p df/emboj00019-0179.pdf

[24] Roberts, W.M., Look, A.T., Roussel, M.F. and Sherr, C.J. (1998) Tandem linkage of human CSF-1 receptor (c-fms) and PDGF receptor genes. Cell, 55, 655-661. doi:10.1016/0092-8674(88)90224-3

[25] Rosenkranz, S., Ikuno, Y., Leong, F.L., Klinghoffer, R.A., Miyake, S., Band, H., et al. (2000) Src family kinases negatively regulate platelet-derived growth factor alpha receptor-dependent signaling and disease progression. Journal of Biological Chemistry, 275, 9620-9627. doi:10.1074/jbc.275.13.9620

[26] Xiong, Y., Song, D., Cai, Y., Yu, W., Yeung, Y.G. and Stanley, E.R. (2011) A CSF-1 receptor phosphotyrosine 559 signaling pathway regulates receptor ubiquitination and tyrosine phosphorylation. Journal of Biological Chemistry, 286, 952-960. doi:10.1074/jbc.275.13.9620

[27] Galvagni, F., Pennacchini, S., Salameh, A., Rocchigiani, M., Neri, F., Orlandini, M., et al. (2010) Endothelial cell adhesion to the extracellular matrix induces c-Src-dependent VEGFR-3 phosphorylation without the activation of the receptor intrinsic kinase activity. Circulation Research, 106, 1839-1848.

doi:10.1161/CIRCRESAHA.109.206326

[28] Lei, H. and Kazlauskas, A. (2009) Growth factors outside of the platelet-derived growth factor (PDGF) family employ reactive oxygen species/Src family kinases to activate PDGF receptor alpha and thereby promote proliferation and survival of cells. Journal of Biological Chemistry, 284, 6329-6336.

http://www.jbc.org/content/284/10/6329.long

[29] Suh, H.S., Kim, M.O. and Lee, S.C. (2005) Inhibition of granulocyte-macrophage colony-stimulating factor signaling and microglial proliferation by anti-CD45RO: Role of Hck tyrosine kinase and phosphatidylinositol 3kinase/Akt. Journal of Immunology, 174, 2712-2719. http://www.jimmunol.org/cgi/pmidlookup?view=long\&p $\underline{\mathrm{mid}}=15728479$

[30] Baran, C.P., Tridandapani, S., Helgason, C.D., Humphries, R.K., Krystal, G. and Marsh, C.B. (2003) The inositol 5'-phosphatase SHIP-1 and the Src kinase Lyn negatively regulate macrophage colony-stimulating factor-induced Akt activity. Journal of Biological Chemistry, 278, 3862838636. doi:10.1074/jbc.M305021200

[31] Rovida, E., Spinelli, E., Sdelci, S., Barbetti, V., Morandi, 
A., Giuntoli, S., et al. (2008) ERK5/BMK1 is indispensable for optimal colony-stimulating factor 1 (CSF-1)-induced proliferation in macrophages in a Src-dependent fashion. Journal of Immunology, 180, 4166-4172. http://www.jimmunol.org/content/180/6/4166.long 